\title{
Hormigón con bajo contenido de cemento y hormigón con sustitución parcial de cemento
}

Prof. Dr. JOSE CALLEJA IETCC

\section{$R E S U M E N$}

Hace algún tiempo se preparó y difundió por el miembro noruego (12) del CEB (Comité Europeo de Hormigón), entre los componentes de la Comisión VIII del mismo (Tecnología y Control de Calidad de Hormigón), una Encuesta sobre los titulos que encabezan este trabajo.

Las respuestas recibidas fueron estudiadas por el firmante del mismo, a petición de dicha Comision VIII del CEB, y el contenido ordenado de dichas respuestas constituye el objeto de este Informe, en el cual se hacen destacar algunas conclusiones de aquélla.

Entre las respuestas mencionadas figuraba la española, la cual corrió, en su momento, a cargo del autor del presente trabajo.

\section{$S U M M A R Y$}

Some time ago an Inquiry concerning the items exposed in the title of this Report was prepared and circulated by the Norwegian member of the CEB/Commission VIII, among the members of this Commission.

The responses received so far have been collected and studied, and the ordered content of them has been exposed in this Report according to the items involved in the Inquiry.

Short general conclusions have been drawn from the mentioned content.

\section{INTRODUCCION}

En la reunión celebrada en Madrid, en Noviembre de 1979, por la Comisión VIII del CEB HORMIGON: "Tecnología y Control de Calidad", se convino en que el tema relativo a las "Propiedades del hormigón con contenido reducido de cemento", o con "Sustituc:ón parcial de cemento", podría ser muy importante en lo que concierne a la calidad, comportamiento, prestaciones y durabilidad de las estructuras de hormigón.

Se convino también en que, en consecuencia, se debería proceder a realizar una Encuesta como base para un Informe acerca de la situación general del tema - si ello fuese posible- o, en otro caso, sobre la situación particular del mismo en distintos países.

El punto de partida para la Encuesta fue la consideración de que el desarrollo y la experiencia ganada en el conocimiento de los materiales y en la técnica del hormigón permiten hoy día la producción de hormigones con menor contenido de cemento y mayores relaciones agua/cemento (a/c), tales que cumplan con unas exigencias dadas, relativas a la resistencia a compresión.

Se mencionó como ejemplo el caso de Noruega, según el cual el empleo de polvo de sílice como sustitutivo de una parte de cemento ha permitido hacer hormigones de $60 \mathrm{MPa}$ y $40 \mathrm{MPa}$ de resistencia a compresión, con relaciones a/c de 0,55 y 0,77 , y con contenidos de cemento de menos de $300 \mathrm{~kg} / \mathrm{m}^{3}$ y $200 \mathrm{~kg} / \mathrm{m}^{3}$, respectivamente. 
Este ejemplo evidentemente se refiere a la sustitución de cemento por otro material, más que a la reducción del contenido de cemento en los respectivos hormigones. Sin embargo, se podría considerar que, al menos de forma indirecta, se consigue al mismo tiempo una reducción de cemento "portland puro", por así decir. Esta observación es importante, ya que explica de antemano ciertas interpretaciones hechas en algunas de las respuestas dadas a la Encuesta.

La razón principal de ésta fue la duda acerca de si las propiedades del hormigón, distintas de su resistencia mecánica —como, por ejemplo, su estabilidad (retracción, expansión...), su durabilidad (resistencia química, resistencia al hielo, corrosión de armaduras...), etc.-, comparadas con las de hormigones usuales de la misma o similar resistencia mecánica se alcanzarían a las m:smas edades cortas, y si se mantendrían junto con la propia resistencia mecánica a edades largas, cuando el contenido de cemento se reduce por sustitución parcial por otro material.

Las cuestiones planteadas en la Encuesta fueron las siguientes:

\section{Práctica común del hormigón}

a) Extensión del empleo de hormigón con contenido reducido de cemento o con sustitución parcial de cemento.

b) Tipos de sustitutivos de cemento utilizados corrientemente.

c) Motivos técnicos y/o económicos para la utilización de sustitutivos del cemento (reducción del costo del cemento, consecución de estructuras con mejor comportamiento).

d) Otros métodos para reducir el contenido de cemento (distintos del empleo de superplastificantes).

\section{Normas nacionales}

a) Tipos de restricciones nacionales y razones para las mismas en cuanto al uso de tales hormigones para estructuras especiales (condiciones de exposición ambiental y de medio, consecuencias de fallos, hormigones de alta resistencia, etc.).

b) Requisitos especiales para el curado y otros aspectos del proceso de ejecución del hormigón.

c) Procedimientos especiales para el control de la resistencia mecánica. (¿Se considera que la relación entre la resistencia potencial convencional y la resistencia in situ es la misma en el caso de los hormigones con menos cemento o con una parte del mismo sustituida, que en el caso de los hormigones tradicionales?).

d) Restricciones en el uso de métodos acelerados para predecir la resistencia final (algunos de los métodos empleados dan normalmente una estimación demasiado alta de la resistencia final de los hormigones en cuestión).

3. Informe de daños

a) Fisuración debida a retracción plástica del hormigón tierno o a retracción restringida o coartada del hormigón endurecido.

b) Daños causados por corrosión de las armaduras de acero (alcalinidad reducida, mayor permeabilidad...). 
c) Daños causados por carbonatación.

d) Daños producidos por la acción del hielo o por otras condiciones climáticas.

4. Hallazgos de la investigación y puntos de vista técnicos y opiniones generales acerca de la durabilidad y del comportamiento a largo plazo

a) Desarrollo de la resistencia en función del tiempo.

Observación: Algunos sustitutivos del cemento pueden dar en los hormigones resistencias a tracción mucho más bajas que las de los hormigones ordinarios de cemento portland de igual resistencia a compresión, pero que no contienen tales sustitutivos, especialmente después de una desecación —véase el punto $4 \mathrm{~b}$ )—.

b) Retracción restringida o coartada.

Observación: El efecto de las relaciones a/c altas y/o del empleo de algunos sustitutivos del cemento es que pueden aumentar la retracción del mortero matriz, dando lugar a una microfisuración interna y a una reducción de la resistencia a la tracción del hormigón - véase el punto $4 \mathrm{a})$ -

c) Retracción plástica (capilar).

Observación: Algunos sustitutivos del cemento extremadamente finos (como, por ejemplo, el polvo de sílice) eliminan la exudación o rezumado del hormigón, haciéndole así más vulnerable a la fisuración por reträcción plástica.

d) Calor de hidratación.

Observación: Se considera, en general, que el uso de sustitutivos puzolánicos hace disminuir el calor de hidratación. No obstante, el polvo de sílice (85 \% de $\mathrm{SiO}_{2}$ ) hace aumentar el calor de hidratación del hormigón cuando reemplaza a una masa equivalente de cemento.

e) Propiedades reológicas.

Observación: Hay razones para creer que cuando se puede hacer hormigón de una determinada categoría resistente especificada, con un contenido de cemento mucho menor que el que ha sido normal hasta ahora, dicho hormigón puede presentar propiedades reológicas totalmente diferentes (relajación, fluencia, alargamiento, fragilidad, etc.). Estas propiedades son extremadamente importantes para estructuras de hormigón de alta resistencia. Si las diferencias existentes son muy grandes, puede ser necesario introducir cambios en las instrucciones para el proyecto del hormigón.

f) Alcalinidad, permeabilidad.

Observación: La reacción puzolánica y/o un bajo contenido de cemento pueden reducir la alcalinidad del hormigón, dando lugar a una pérdida de protección contra la corrosión del acero y la carbonatación. El efecto aumentará si el hormigón se hace con una relación a/c más alta (mayor permeabilidad) que la normal para un hormigón ordinario de igual resistencia.

g) Resistencia al hielo.

Observación: Si la utilización de ciertos sustitutivos del cemento permite hacer un hormigón de una categoría resistente especificada, con una relación a/c mayor que la del hormigón ordinario, la resistencia al hielo disminuirá. 
h) Resistencia a la abrasión.

Observación: Se considera que algunos sustitutivos del cemento mejoran la resistencia a la abrasión de la superficie del hormigón, al eliminar la exudación y la segregación.

i) Resistencia a los sulfatos y a los ácidos.

Observación: Parece estar reconocido que los sustitutivos del cemento con buenas propiedades puzolánicas garantizan una mayor durabilidad contra ataques por sulfatos solubles y ácidos débiles (para valores de la relación a/c iguales o muy similares).

j) Otras propiedades o aspectos.

La Encuesta estaba dirigida a todos los miembros de la Comisión VIII del CEB y a otras personas interesadas, por el Ing. Randulf JOHANSEN, Jefe de Construcción y Control en el Instituto de Investigación del Hormigón, en N-7034 Trondheim - NTH- Noruega.

En su condición de "persona interesada", el autor de este Informe envió en Enero de 1981 a los mencionados nombre y dirección algunos comentarios extensos, relativos a los puntos de la Encuesta.

Mucho después, en Octubre de 1981, el autor recibio del Prof. Dr. Ing. Helmut WEIGLER, Institut für Massivbau, Technische Hochschule, Darmstadt, RFA, miembro de la Comisión VIII/CEB, algunas (pocas) respuestas a la Encuesta, con la invitación de estudiarlas y resumir las conclusiones derivadas de ellas. Circunstancias personales impidieron al autor hacerlo con anterioridad, y si bien se llevó a cabo en su momento mediante un Informe en inglés para el CEB, se vuelve a hacer aquí y ahora en castellano para los lectores hispanohablantes.

\section{COMEntarios a LOS PUNTOS De LA ENCUESTa}

De los 19 países miembros representados en la Comisión VIII del CEB, sólo de 8 se recibieron respuestas a la Encuesta, a saber: de Alemania RF', Austria, Dinamarca, España, Grecia, Jordania, Polonia y CEMBUREAU (la Asociación Europea del Cemento).

Las respuestas recibidas consistieron, más o menos, en comentarios generales a la Encuesta en conjunto, y/o en comentarios particulares acerca de algunos puntos de la misma.

\subsection{Comentarios generales a la encuesta}

Tal vez el comentario más significativo al punto $1 \multimap$ pero también a la Encuesta en su conjunto- fue el del CEMBUREAU, expuesto por su Director, quien al mismo tiempo es Presidente de la Comisión Técnica CEMENTO CEN/CT-51 (1).

Según él, es necesario aclarar el significado de la palabra "cemento", habiendo dos respuestas posibles para la correspondiente pregunta: 1) o bien se designa sólo como cemento a la mezcla clásica de clínker y retardador de fraguado - generalmente una o varias formas de sulfato cálcico-; 2) o bien se puede llamar también cementos a distintas mezclas de clínker y retardador con otros varios constituyentes secundarios, tales como las escorias de horno alto, las puzolanas, las cenizas volantes, los fillers... en proporciones diferentes, con tal de que dichas mezclas se hagan en fabricas de cemento, cumplan las nor- 
mas nacionales y/o internacionales para los cementos correspondientes y estén sujetas a controles internos de calidad de los fabricantes, y a veces externos de los usuarios.

La respuesta 1) parece haber sido adoptada en los puntos de la Encuesta, mientras que la respuesta 2) es la corriente en muchos países del mundo, y es también la adoptada por el CEN/TC-51. Un tercer caso sería el de los países que tienen en cuenta los llamados "cementos especiales", no siempre incluidos en las normas.

Los comentarios procedentes de Dinamarca (2) y de España (3) estuvieron en completo acuerdo con el punto de vista del CEMBUREAU. Como se verá después, también Alemania $R F$ coincidió en ello — véase el punto 2.2 .4 j)—.

En el caso de España (3) se hizo una precisión en el sentido de diferenciar la "reducción" del consumo de cemento en el hormigón, bien mezclando materiales que sustituyen al cemento en las obras, o bien usándolos como adición al clínker en las fábricas de cemento. En la primera solución existe el riesgo de la falta de uniformdad en las proporciones de mezcla, y de homogeneidad y constancia de calidad y control del hormigón resultante, mientras que en la solución segunda no se dan esos inconvenientes, puesto que permite en cada caso establecer proporciones óptimas en las mezclas, dependiendo de la naturaleza, calidad y características de los materiales implicados.

Otro aspecto general comentado fue el relativo al ejemplo noruego del polvo de sílice utilizado como sustitutivo del cemento. El punto de vista español (3) consideró que dicho ejemplo era muy específico y que, por lo tanto, sus aspectos particulares no podían extenderse al caso de cualquier otro material clásico de adición al clínker en las fábricas de cemento, o al cemento en las obras, si bien el polvo de sílice podría ser comparado en cierta medida con algunas cenizas volantes respecto de la finura, y con el kieselguhr en lo que concierne a la composición.

Un punto de vista alemán (4) consideró que con el polvo de sílice, así como con las cenizas volantes, es posible obtener resistencias bastante altas, particularmente mediante tratamientos hidrotérmicos, pero que debía prestarse atención a la reducción de la alcalinidad y a un posible descenso del $\mathrm{pH}$, en cuanto al riesgo de pérdida de protección contra la corrosión de las armaduras se refiere, así como a la necesidad de contrarrestar el potencial de corrosión mediante otras acciones, y en particular utilizando otras adiciones o aditivos para el hormigón.

Una opinión semejante fue mantenida por Austria (5), considerando que las mejoras en la fabricación de cemento y la caracterización de los hormigones por su resistencia a compresión han favorecido la consecución de hormigones con relaciones agua/cemento más altas, suficientemente resistentes, pero con resultados posiblemente desfavorables para otros aspectos del hormigón, tales como la carbonatación (corrosión), la resistencia al hielo, la penetración de iones cloro (y, en general, de sales), etc. En consecuencia, la tendencia austriaca se dirige a utilizar mayores contenidos de cemento, a fin de hacer hormigones exentos de problemas. En este sentido la opinión austriaca — como la alemana (6) - recomienda observar los contenidos minimos de cemento y las máximas relaciones a/c prescritos en las normas para hormigón; y que sólo cuando se cumplen tales requisitos se considera posible añadir hasta 25 por ciento como máximo - referido al peso del cemento- de cenizas volantes para aumentar la trabajabilidad, con tal de que el propio cemento no contenga previamente ninguna otra adición (es decir, cuando el cemento implicado es un cemento portland "puro"). Esta puntualización es importante, ya que realza la significación del comentario del CEMBUREAU (1). Es evidente que el punto de vista austriaco 
acepta condicionalmente una adición de cenizas volantes, pero no una sustitución o una reducción del contenido verdadero de cemento en el hormigón.

Además, la opinión alemana fue que una posible "sustitución" del contenido de cemento en el hormigón armado estaría limitada a la diferencia entre el contenido mínimo de 240 $\mathrm{kg} / \mathrm{m}^{3}$ y el contenido necesario para alcanzar la resistencia requerida a los 28 días (como máximo, 30 por ciento del peso del cemento) (6). Hay que aclarar que esto no parece ser una verdadera sustitución, sino una adición de otros materiales al contenido mínimo de cemento portland de acuerdo con las normas $\left(240 \mathrm{~kg} / \mathrm{m}^{3}\right)$, en proporción tal que la resistencia exigida a los 28 días se consiga por este medio, en lugar de utilizar mayores contenidos de cemento portland "puro". Esto se ha hecho desde hace unos 12 años, principalmente en las plantas preparadoras de hormigón (6).

En una dirección semejante apuntaron otras informaciones alemanas, según las cuales sólo se está llevando a cabo una sustitución parcial de cemento portland por cenizas de carbón mineral pulverizadas, pero no por polvo de sílice; una explicación es que la composición de las cenizas es más o menos semejante a la de algunos componentes del cemento portland, mientras que la del polvo de sílice no lo es (6).

Por otra parte, la reducción de cemento en la medida indicada en la Encuesta no está permitida en Alemania (6), y no es probable que en un futuro próximo las especificaciones alemanas puedan admitir una sustitución sustancial de cemento por otros materiales y, en consecuencia, que tal sustitución sea adoptada como práctica común en el hormigón estructural. No obstante, es posible que un conocimiento y una experiencia más amplios puedan tal vez cambiar la situación en un futuro lejano (4).

Una observación final por parte de Alemania, que subraya alguno de los puntos de vista ya expuestos (1) (3) (5), es que la Norma DIN 1164 - como otras muchas normas nacionales-, considera como sustitutivos del cemento portland a aquellos otros cementos que contienen constituyentes semejantes a los utilizados en las obras (6).

\subsection{Comentarios particulares a la encuesta}

\subsubsection{Comentarios al punto 1: Práctica ordinaria}

a) En lo que se refiere a la extensión del uso de hormigón con contenido reducido de cemento o con sustitución parcial de cemento, las respuestas de tres países difirieron ampliamente, siendo los valores señalados de 2 por ciento (2), 20-35 por ciento (6) y más de 80 por ciento (7) — cifras referidas a la cantidad total de hormigón-.

b) En cuanto a los tipos de sustitutivos más corrientemente usados, las respuestas de cinco países mencionaron: i) las cenizas volantes y el polvo de sílice (2); ii) las cenizas volantes de carbón mineral que cumplen algunos requisitos relativos a composición, propiedades y uniformidad, y cuya adecuación está certificada (6), y no sólo como sustitutivo del cemento, sino también como reemplazo de una parte del árido fino en el hoimigón (8); iii) las puzolanas (7); y iv), materiales activos puzolánicos naturales o artificiales (cenizas volantes o arcillas activadas térmicamente), o escorias básicas granuladas de horno alto, todos ellos como sustitutivos de clinker en fábricas de cemento y muy raramente como sustitutivos del cemento en plantas de hormigón o en obras (3).

c) En lo relativo a los motivos técnicos y/o económicos para utilizar sustitutivos del cemento (reducción del costo del cemento, consecución de estructuras con mejor com- 
portamiento), las respuestas de cinco países invocaron unánımemente razones económicas: i) bajar los costos de los materiales en general (6) y del cemento (7) o del hormigón (8) en particular; ii) ahorrar clínker —energía térmica y eléctrica (3) - y/o cemento (2) (3); iii) aumentar la producción (7); iv) utilizar subproductos y materiales de desecho recuperando su masa y energía potencial (3). También invocaron razones técnicas: i) mejorar la trabajabilidad y la consistencia del hormigón fresco, y la impermeabilidad del hormigón endurecido, rebajando la relación a/c (6) (2); ii) mejorar la capacidad de bombeo y el hormigonado bajo agua mediante un hormigón fresco más coherente (2); iii) aumentar la densidad del hormigón y mejorar sus propiedades aislantes —en cada caso- (8); iv) mejorar la resistencia del hormigón al ataque por sulfatos y la durabilidad en general utilizando tipos de cementos para usos especiales (3) (7). Finalmente se adujeron razones ecológicas basadas en la supresión de acumulaciones de desechos de cenizas (3) (8) y de escorias (3).

d) Las respuestas a otros métodos para rebajar el contenido de cemento (distintos del uso de plastificantes ordinarios), pertenecientes a cinco países, señalaron que, o bien no existen tales métodos (6), o que éstos pueden consistir en un elevado control en el nivel de producción y en una reducción de los finos del hormigón (7); o en procesos de curado térmico de hormigones con un contenido elevado de cenizas volantes que permiten una disminución del contenido de cemento (8). Dos países mencionaron el uso de superplastificantes (2) (3), particularmente cuando se utilizan áridos de baja calidad, lo cual implica un aumento de la exigencia de agua (2). También se hizo una observación relativa a la reducción del contenido de cemento utilizando, para el mismo hormigón, cementos de mayor categoría resistente en cantidades menores, (3). Se considera que esta práctica es perniciosa, ya que se pueden dañar otras propiedades, bien sean del hormigón fresco o del hormigón endurecido, a través del comportamiento reológico del primero, o de los parámetros físicos y de la durabilidad del segundo, respectivamente. Esto es válido, tanto si los cementos pertenecen al mismo tipo y clase como si no (por ejemplo, cuando cementos puzolánicos o siderúrgicos se sustituyen por cementos portland). Se deja aparte otro aspecto económico, cual es el de que los cementos sustituyentes de superior categoría resistente son en general más costosos que los sustituidos de categoría resistente inferior, con lo cual poco - si algose gana en precio, cuando no resulta perjudicial la sustitución por tal concepto, a lo que hay que añadir los restantes inconvenientes ya señalados.

\subsubsection{Comentarios al punto 2: Normas nacionales}

a) En cuanto a los tipos y motivos para las restricciones nacionales en el uso de tales hormigones (con menor contenido de cemento) para estructuras especiales (condiciones de exposición ambiental, consecuencias de fallos, hormigones de alta resistencia, etc.), las respuestas recibidas de cinco países fueron diferentes. Algunos de ellos no reconocen restricciones (3) (6) (7), o fijan el límite en un 30 por ciento de ceniza volante (referido al peso del cemento) a fin de evitar aumentos de la corrosión de las armaduras, si bien no se ha confirmado una corrosión mayor con contenidos de ceniza hasta 150-300 por ciento del peso del cemento (8). En otros casos los límites superiores para la ceniza volante y para el polvo de sílice son $60-70$ por ciento y 8-10 por ciento respectivamente (2). Algunos Códigos Prácticos no contienen sino muy pocas directrices a este respecto (2) (3). No obstante, especifican un valor máximo para la relación a/c (2) calculada sobre la base del contenido de cemento exclusivamente, y un contenido de cemento mínimo (6) (3). En este sentido se piensa que los hormigones demasiado pobres son peligrosos para estructuras expuestas a la intemperie o a condiciones de servicio particulares - heladas- (6). Si bien se están discutiendo en algunos casos determinadas especificaciones y requisitos más drásticos (6), en otros 
casos no se toman en consideración requisitos especiales para el curado, el control, etc. (2). Finalmente, en algunas circunstancias existen indicaciones técnicas más o menos obligatorias o con carácter de recomendación, respecto del uso de determinados tipos de cemento para hormigones especiales, estructuras específicas y en medios ambientales particulares (3).

b) Por lo que respecta a los requisitos especiales parael curado y otros aspectos del proceso de ejecución, cinco respuestas pusieron de relieve la falta de ellos en tres países (2) (3) (6), si bien en uno se reconoció la necesidad de un curado prolongado de los hormigones que contengan puzolanas o adiciones hidráulicas latentes -escorias- (6), así como en el hormigonado en invierno (7). El hormigón que contenga cenizas deba cumplir con las normas establecidas para el mismo, según se hizo constar en otro caso (8).

c) En lo concerniente a procedimientos especiales de control para la resistencia ( $\mathrm{se}$ considera que la relación entre la resistencia potencial convencional y la resistencia in situ es la misma que en el caso de los hormigones tradicionales?), dos respuestas mostraron que no existen procedimientos especiales de control (3) (6), y que probablemente las relaciones mencionadas no son las mismas, si bien este punto no ha sido aclarado del todo (6).

d) Una respuesta a la cuestión sobre las restricciones en el uso de los métodos acelerados para predecir la resistencia final (algunos de los métodos empleados dan normalmente una estimación demasiado alta de la resistencia final de tales hormigones - con menor dosificación de cemento o con sustitución parcial del mismo-), señaló que, en general, no existen semejantes métodos normalizados, y que los recomendados muy raramente se utilizan, ni para hormigones ordinarios ni para hormigones con contenido reducido de cemento; se aplican principalmente para controlar la regularidad de la calidad - resistencia- de la producción de cemento en las fábricas de este material. De todos modos sus resultados son más dignos de confianza en el caso de los cementos portland que en el caso de los cementos parcialmente sustituidos que contienen adiciones; aplicados a hormigones, parecen causar efectos más intensos y destacados en el caso de hormigones ricos que en el caso de hormigones pobres (3).

\subsubsection{Comentarios al punto 3: Informe de daños}

a) En cuanto a la fisuración debida a la retracción plástica del hormigón endurecido, cinco respuestas pusieron de relieve: i) que han sido señaladas fisuraciones debidas a retracción plástica en hormigones que contenían cantidades excesivas de cenizas volantes o de poilvo de sílice (2); ii) que en análogas condiciones se han observado también fisuraciones en hormigones endurecidos (6); iii) que la fisuración detectada en hormigones que contienen puzolanas ha sido atribuida a otros factores; y iv) que se ha apreciado una fuerte retracción plástica (de "acomodación") en hormigones hechos con cementos con "falsas puzolanas" (no auténticas), debido a curvas granulométricas anormales de los cementos que las contenían, a unas exigencias de agua excesivas, a una escasa retención de agua y a una gran exudación (3). Finalmente, algunos resultados contradictorios indicaron que ciertos hormigones con un contenido elevado de cenizas volantes se comportan mejor que algunos hormigones tradicionales en lo que se refiere a la fisuración (8).

b) Con respecto a los daños causados por corrosión del acero de las armaduras (alcalinidad reducida, mayor permeabilidad), cinco respuestas indicaron: i) que no se han señalado daños hasta el momento (2) (7) o en el término de dos años (8) en hormigones 
armados, con contenidos altos de cenizas volantes; y ii) que estructuras hechas de hormigón con contenido bajo de cemento y alto de adiciones han sufriüo frecuentemente daños por corrosión dèl acero de las armaduras, cuando han estado expuestos a la intemperie, siendo la baja dosificación de las mezclas una de las causas de la reducción de la alcalinidad y del aumento de la permeabilidad de los mismos, particularmente ayudada por un curado inadecuado (6). Parcialmente de acuerdo con este comentario y según algunas otras consideraciones difundidas, otro punto de vista (3) indicó que no son probables grandes diferencias entre cementos portland y cementos que contienen sustitutivos, en lo que se refiere a la protección de las armaduras, en circunstancias ordinarias; pero que tales diferencias pueden existir en el caso de hormigones porosos y permeables sometidos a acciones agresivas externas. Algunas otras consideraciones llevan a pensar que no hay razones para sospechar que los cementos que contienen sustitutivos son siempre responsables de la corrosión de las armaduras en el caso de hormigones bien proyectados, ejecutados y curados (3).

c) En lo concerniente a daños causados por carbonatación las cinco respuestas indicaron: i) que no se han registrado hasta la fecha (2) (7) o al cabo de dos años (8); ii) que se ha observado una mayor profundidad de carbonatación en elementos de hormigón con bajo contenido de cemento y alto contenido de adiciones, si bien estas observaciones deben ser evaluadas con más detenimiento (6); iii) que, sobre la base de consideraciones teóricas, es previsible que la carbonatación del hormigón afecte más a hormigones hechos con cemento portland que a los hechos con cementos siderúrgicos o puzolánicos (3); y iv) que la porosidad y la permeabilidad al aire del hormigón, principalmente en la superficie del mismo, evidentemente tienen una gran influencia y, por otra parte, que es necesario diferenciar entre profundidad de carbonatación y carbonatación total.

d) En cuanto a los daños causados por la acción del hielo o por otras condiciones climáticas, las cinco respuestas señalaron: i) que no se habían registrado daños (2) (7); ii) que no se habían observado diferencias con respecto a los hormigones ordinarios (8); y iii) que se había apreciado una menor resistencia al hielo en elementos de hormigón pobres en cemento y ricos en adiciones, si bien este punto debería ser considerado con mayor detenimiento (6).

2.2.4. Comentarios al punto 4: Hallazgos de la investigación y puntos de vista técnicos $y$ opiniones acerca de la durabilidad $y$ del comportamiento a largo plazo

a) Con respecto al desarrollo de la resistencia en función del tiempo, habida cuenta de que algunos cementos con sustitutivos pueden dar hormigones con resistencias a tracción mucho más bajas que las de los hormigones ordinarios de cemento portland, especialmente después de una desecación - véase también el punto siguiente $4 \mathrm{~b}$ )-, contestaron cinco países, así: i) la resistencia a compresión alcanzada a edades largas (tres meses) es mayor en hormigones (cementos) que contienen cenizas volantes y polvo de sílice que en hormigones sin adiciones, ocurriendo lo contrario a edades cortas $(2)$; ii) en el caso de una sustitución del 25 por ciento de cemento por escoria la diferencia es menor a edades largas y casi desaparece al cabo de tres meses (6); iii) este plazo se reduce a 28 días según otros resultados (7). En general no se hicieron investigaciones o comentarios relativos a la resistencia a la tracción. Sin embargo, se hizo una observación en el sentido de que en algunos casos la relación entre las resistencias a tracción y a compresión podía ser mayor que la usual, incluso con cementos que contuviesen adiciones activas - puzolanas-, particularmente si se utilizaran en combinación con algunos superplastificantes (3). 
b) En cuanto a la retracción restringida, teniendo en cuenta que el efecto de una relación a/c alta y/o el uso de algunos sustitutivos del cemento pueden aumentar la retracción de la matriz de mortero, dando lugar a microfisuras internas y a una disminución de la resistencia a la tracción del hormigón -véase también el punto precedente 4 a)-, se expusieron pocas opiniones, a causa de una falta de investigación al respecto (2) (7). En otro caso se consideró que el hormigón con un alto contenido de cenizas volantes requiere una cantidad de agua mayor, siendo la relación a/c 0,8-2,0, pero si esta relación es la adecuada no se observa microfisuración, con tal de que se mantenga un ambiente suficientemente húmedo - condiciones de curado adecuadas(8). Algunas otras consideraciones (3) reconocieron que la microfisuarción interna es previsible, por una mayor retracción y también por una menor resistencia a la tracción, en hormigones que contengan adiciones, particularmente si su exigencia de agua es grande y su capacidad de retención de agua escasa, pero que los cementos que contienen tales adiciones pueden conferir una gran deformabilidad a los hormigones, de tal manera que se puede establecer una compensación o compromiso entre retracción, resistencia intrínseca a la tracción y deformabilidad - módulo de elasticidad-, siendo los tres parámetros complejos y estando estrechamente relacionados entre si. Bajo la influencia simultánea de la retracción y de la deformabilidad, la pérdida de resistencia a la tracción puede ser el resultado de la microfisuración, o la microfisuración puede ser el resultado de una baja resistencia a la tracción, previamente operante. Así se puede esperar una mayor o menor capacidad para la fisuración interna en cualesquiera hormigones, dependiendo de los parámetros señalados, de sus mutuas relaciones y de los factores que influyen en todos ellos. De cualquier forma, un medio de evitar o atenuar la influencia negativa de algunos de dichos factores, particularmente de una gran exigencia de agua y de una capacidad de retención baja, es decir, de relaciones a/c elevadas, requeridas por algunos cementos y hormigones que contienen adiciones, puede ser el uso de superplastificantes (3).

c) Respecto de la retracción plástica (capilar), teniendo en cuenta que algunos sustitutivos del cemento extraordinariamente finos (por ejemplo, el polvo de sílice) eliminan la exudación del hormigón, haciendole así más vulnerable a la fisuración por retracción plástica, una de las opiniones emitidas consideró, como una observación de carácter general, que la retracción de secado y/o la química mientras el hormigón está aún fresco es mayor en hormigones con sustitutivos del cemento que en hormigones sin ellos, particularmente si el sustitutivo es polvo de sílice (2) (8). Otro punto de vista consideró que no está del todo claro si la eliminación de la exudación causa siempre una mayor fisuración por retracción plástica (de "acomodación"), o si algunas facilidades para là exudación pueden ayudar algunas veces a evitarla, dependiendo de las circunstancias - condiciones ambientales y de curado, granulometría de los cementos y de los sustitutivos, etc.-. Por ejemplo, algunos elementos estructurales de gran superficie y pequeño espesor, sometidos a un fuerte viento seco y caliente en un ambiente cálido y de baja humedad relativa, experimentan una desecación intensa y rápida de su hormigón, favorecida por la exudación, de tal manera que pueden tener lugar una retracción y una fisuración plásticas, ya que la "acomodación" del horhormigón fresco puede no producirse del todo y en el debido tiempo. Pero, en condiciones opuestas -o simplemente en condiciones ordinarias-, la capa de agua formada en la superficie por exudación se evapora mucho más lentamente, actúa como una cubierta de curado húmedo y permite que tenga lugar la "acomodación" sin retracción y fisuración plásticas, o en todo caso siendo estas mucho menores. La composición -curva-granulométrica, bien sea del cemento que contiene sustitutivos del clínker, o bien del sustitutivo del cemento añadido en la hormigonera, pueden tener 
una gran influencia en la exigencia y en la retención de agua y en la exudación, y consecuentemente en la retracción y en la fisuración plásticas (3).

d) En lo que concierne al calor de hidratación, teniendo en cuenta que el empleo de sustitutivos puzolánicos va generalmente unido a una disminución del mismo, y que, sin embargo, el polvo de sílice con 85 por ciento de $\mathrm{SiO}_{2}$ aumenta el calor de hidratación del hormigón cuando reemplaza a una masa equivalente de cemento, las respuestas mostraron: i) que, de hecho, se ha observado alguna disminución de dicho calor (7); ii) que las curvas de calor de hidratación de los hormigones con y sin cenizas volantes son muy similares, con tal de que las curvas de desarrollo de las resistencias de ambos sean idénticas, mientras que el hormigón con polvo de sílice muestra un pico desfavorable en la curva del calor de hidratación a las 10-15 horas de endurecimiento (2); y iii) que se ha ensayado y observado que el calor de hidratación depende de la temperatura inicial del hormigón, de la calidad del cemento, de los contenidos de cemento y de ceniza y de las relaciones agua/cemento y agua/(cemento + ceniza) (8). Finalmente, se expusieron algunas precisiones (3) para explicar con mayor detalle el hecho de que los sustitutivos puzolánicos disminuyen el calor de hidratación de los cementos y de los hormigones que los contienen, particularmente a cortas edades de la hidratación, y más o menos proporcionalmente a la cantidad de clínker reemplazado por el sustitutivo. De tal manera que el plazo al cual la sustitución de clínker por puzolanas produce una disminución del calor de hidratación de los cementos depende de la naturaleza de ambos, de sus proporciones relativas y de la finura de los cementos resultantes; todo ello en la forma que es fácilmente previsible. La acción puzolánica es tal que a partir de un tiempo dado es posible que en ciertas condiciones el calor de hidratación de un cemento puzolánico sea mayor que el de un cemento portland equivalente, comparable con él. No obstante, pueden existir grandes diferencias en lo que se refiere a la velocidad o ritmo de desprendimiento del calor en ambos casos. En cuanto al polvo de sílice, habida cuenta de su naturaleza y actividad, es de prever que el plazo al cual los cementos que lo contienen den un calor de hidratación mayor que el correspondiente a cementos portland comparables, no sustituidos, sea mucho más corto; y también que sea mucho más corto que el de los cementos, asimismo equivalentes, pero que contengan otros materiales puzolánicos no tan activos. Esto podría inducir a considerar la posibilidad de aprovechar estos hechos en la fabricación de productos silicocalcáreos basada en tratamientos higrotérmicos (3).

e) En lo que respecta a las propiedades reológicas, y habida cuenta de que hay una razón para creer que cuando se produce un hormigón de una determinada calidad resistente con un contenido de cemento mucho menor que el ordinariamente normal, dicho hormigón puede presentar propiedades reológicas muy distintas (relajación, fluencia, deformación, fragilidad, etc.); de que estas propiedades son extremadamente importantes en estructuras hechas con hormigón de alta resistencia; y de que si existen grandes diferencias éstas pueden ex gir un cambio en el proyecto del hormigón, las respuestas mostraron: i) que el hormigón de alta resistencia hecho con polvo de sílice presenta una mayor fragilidad y una menor relación entre las resistencias a tracción y compresión que el hormigón convencional - véase el punto 2.2.4. a) - (2); ii) que la presentación de este aspecto en la Encuesta fue correcta y su contenido absolutamente razonable —véase la última parte del punto 2.2.1. d) - (3); y iii), que un buen (o normal) comportamiento reológico del hormigón requiere un contenido mínimo de matriz de pasta de cemento en el hormigón, a ser posible con una relación a/c óptima, considerando no sólo la resistencia final del hormigón endurecido, sino también las propiedades reológicas del hormigón fresco. En este aspecto la influencia de los superplastificantes debe ser tenida en cuenta una vez más, incluso —o precisamente- en las instrucciones para el proyecto (3). 
f) Con respecto a la alcalinidad y a la permeabilidad, considerando que la reacción puzolánica y/o el empleo de un contenido bajo de cemento pueden reducir la alcalinidad del hormigón, ocasionando con ello una pérdida de protección contra la corrosión del acero y contra la carbonatación; y que este efecto aumenta si el hormigón se hace con una relación a/c mayor (mayor permeabilidad) que la normal para un hormigón ordinario de la misma resistencia, las respuestas fueron: i) que las investigaciones acerca de la composición química del líquido de los poros en el hormigón endurecido han puesto de manifiesto que la adic:ón de cantidades incluso grandes de cenizas volantes al hormigón no modifican significativamente el valor del $\mathrm{pH}$ (2) (8), no siendo aplicable este resultado al polvo de sílice (2); ii) que no se ha observado una notable diferencia con respecto al comportamiento frente a la carbonatación, entre morteros y hormigones con y sin cenizas de carbón pulverizado, si éstos se comparan a igualdad de resistencia (6) ; iii) que este aspecto está íntimamente relacionado con los aspectos tratados en los puntos 2.2.3. b) y 2.2.3. c), y que en condiciones ordinarias no parece probable que exista una diferencia grande entre el grado de protección de las armaduras conferido por los cementos portland "puros" más protectores y los cementos portland con sustitutivos, potencialmente menos protectores, al menos en el caso de hormigones bien proyectados y ejecutados, si bien puede haber diferencias en condiciones especiales, como por ejemplo en el caso de hormigones defectuosos, porosos y permeables, expuestos a acciones y medios agresivos externos, como generalmente ha podido ser observado hasta ahora (3); iv) que la porosidad y la permeabilidad, y sus opuestas: la densidad y la compacidad, y la impermeabilidad e impenetrabilidad, son factores que contribuyen muchísimo en uno u otro sentido a la corrosión, de modo que un hormigón muy compacto e impermeable hecho con un cemento menos protector es capaz de evitar, atenuar o retardar la corrosión mucho más eficazmente que otro hormigón menos permeable y compacto, aunque hecho con un cemento más protector (3) ; v) que esto mismo es válido en lo que se refiere al espesor de los recubrimientos de las armaduras, de manera que, en general, la compacidad e impermeabilidad y una buena adherencia del recubrimiento son mucho más importantes que su espesor, desde el punto de vista de la protección de la armadura, como se ha podido observar (3); y vi), que hay que prever que la carbonatación afecta principalmente a la pasta de cemento y más particularmente a la cal de hidrólisis, menos a los aluminatos hidratados y mucho menos aún a los silicatos hidratados, y que en este aspecto es asimismo previsible que la carbonatación pueda afectar más a los hormigones con cementos portland "puros" que a los que contienen cementos con sustitutivos, a contenidos iguales de clínker, y tanto más cuanto mayor sea el contenido de cemento del hormigón, en cualquier caso. De este modo los hormigones hechos con cementos que contuviesen adiciones serían menos carbonatables que los hechos con cementos portland "puros", lo cual puede ser beneficioso o perjudicial según los espectos que se consideren en cada caso (3).

g) En lo que se refiere a la resistencia al hielo, y teniendo en cuenta que si el uso de ciertos sustitutivos del cemento permite hacer hormigones de una categoría resistente especificada con una relación a/c mayor que la de los hormigones ordinarios, se puede disminuir la resistencia a las heladas, las respuestas expresaron: i) que en el caso del hormigón con cenizas volantes puede haber dificultades en obtener la cantidad adecuada de aire incluido (2); ii) que los morteros con cenizas de carbón pulverizadas expuestos a ciclos de hielo y deshielo no presentan un comportamiento significativamente distinto, y en particular no peor que el de los morteros que no las contienen (6); iii) que se ha observado una buena resistencia al hielo en hormigones ligeros con un contenido elevado de cenizas volantes, cuando el árido ligero fue suficientemente resistente a la helada (8); y iv), que en cualquier caso se puede evitar la disminución de la resistencia al hielo mediante el uso de agentes inclusores de 
aire combinados o no con superplastificantes, lo cual permite rebajar la relación a/c e impartir al hormigón una mayor resistencia intrínseca a la acción del hielo (3).

h) En lo concerniente a la resistencia a la abrasión, y tomando en consideración el hecho de que al eliminar la exudación y la segregación algunos sustitutivos del cemento pueden aumentar la resistencia a la abrasión de las superficies de hormigón, las respuestas señalaron una falta de resultados experimentales (2) (7) (8), suponiéndose que la resistencia a la abrasión probablemente es menor que en el caso de los hormigones tradicionales o con bajos contenidos de cenizas volantes (8). Finalmente, se sugirió que podría ser así en el caso de sustitutivos que reducen la exudación, pero que surge la duda en el caso de que los sustitutivos la aumenten -véase también los puntos 2.2.3. a) y 2.2.4. c)-

i) Con respecto a la resistencia a los sulfatos y a los ácidos, habida cuenta de que parece estar reconocido que los sustitutivos del cemento con buenas propiedades puzolánicas garantizan un mayor grado de durabilidad frente a sulfatos solubles y ácidos débiles (para un mismo nivel de valores de la relación a/c) las respuestas fueron: i) que cuando las puzolanas tales como las cenizas volantes y el polvo de sílice se emplean para aumentar la densidad del hormigón, parece que se consigue también una mejor resistencia frente al ataque de los sulfatos y de otros agresivos químicos (2); ii) que, como regla general, una sustitución parcial de cemento portland por ceniza pulverizada de carbón aumenta la resistencia de los hormigones a los sulfatos, siendo tal aumento mayor con cementos ricos en $\mathrm{C}_{3} \mathrm{~A}$ y relativamente pequeño con cementos de bajo contenido de $\mathrm{C}_{3} \mathrm{~A}$; iii) que una sus'itución del 50 por ciento de cemento portland por ceniza confiere al hormigón la máxima resistencia frente a sulfatos (6); iv) que, además, la resistencia química en gəneral, no solamente frente a sulfatos y ácidos débiles, sino también frente a aguas puras, blandas y carbónicas agresivas, se le confiere al hormigón mediante buenos materiales puzolánicos (3); y v), que, en lo que se refiere al ataque por agua de mar, los hormigones que contienen escorias de horno alto son mucho más resistentes, con tal de que el contenido de escoria sea relativamente alto - no menor de 65-70 por ciento- (3).

j) Con relación a otras propiedades o aspećos las respuestas consideraron: i) que, en general, las adiciones de puzolanas artificiales tales como cenizas volantes y polvo de sílice se pueden utilizar para mejorar la calidad del hormigón, pero que también pueden ser perjurdiciales si su utilización implica aumentos de la relación a/c hasta niveles que ya no proporcionen la densidad necesaria al hormigón (2); ii) que el coeficiente de conductividad térmica de hormigones que contienen grandes cantidades de cenizas volantes ha resultado ser mucho más bajo (8); iii) que se debe investigar a fondo el efecto de los superplastificantes en la relación entre las resistencias a tracción y compresión, y en las retracciones plástica, hidráulica y restringida, así como en la microfisuración (3) (6); y iv), que las posibilidades fisicoquímicas y técnicas de la fabricación industrial de productos silicocalcáreos y cementicios mediante tratamientos higrotérmicos constituyen otro aspec $` o$ en el cual valdría asimismo la pena una investigación sistemática (3), como también sobre la influencia del polvo de sílice en la reacción árido-álcalis cuando haya que emplear áridos reactivos con cementos portland de contenido normal o alto de álcalis, lo cual se debe evitar en lo posible (3). Finalmente, como una especie de resumen de los aspectos relativos a los sustitutivos y a las adjciones para los cementos, al modo de añadirlos (al cemento en las fábricas o al hormigón en las obras), y a los efectos probables correspondientes, se considera conveniente exponer las siguientes opiniones generales que se manifestaron en (6): "Cuando las adiciones que participan en las reacciones de hidratación, alterando con ello el fraguado y el endurecimiento del hormigón, se añaden en la hormigonera, pue- 
den ejercer una influencia dañina sobre las propiedades del hormigón fresco y :ambién del hormigón endurecido. Esta influencia puede ser diferente para distintos tipos y clases de cementos". "Por lo tanto, es necesario armonizar la adición con el cemento en cada caso". "Sin embargo, esto no se puede garantizar cuando la adición se hace en la hormigonera". "La adición en la hormigonera implica riesgos comparada con el empleo de cementos compuestos producidos en una fábrica de cemento". "Hasta ahora el uso creciente de adiciones al hormigón se ha considerado en primer lugar desde el punto de vista de las resistencias a compresión de hormigones equivalentes a 28 días". "No obstante, al hacerlo así no se garantiza también automáticamente la durabilidad de las estructuras". "Las relaciones que serían imprescindibles para ello no parecen estar todavía suficientemente claras". "Actualmente se hacen esfuerzos en la construcción en hormigón para mantener las reservas necesarias para una durabilidad reforzada". "A este respecto, entre las medidas de tipo estructural que se pueden adoptar, se discute la de aumentar el contenido mínimo de cemento a unos $300 \mathrm{~kg} / \mathrm{m}^{3}$, en estructuras que hayan de estar expuestas a la intemperie".

\section{OTROS COMENTARIOS}

Una respuesta posterior y tardía por parte de Grecia (9) puso de relieve el comienzo de una investigación sobre el uso de cenizas volantes de lignito griegas, bien añadidas en la hormigonera o molidas conjuntamente con el clínker, en proporciones de 0,15 y 30 por ciento. Se estudian o estudiarán parámetros tales como resistencias, retracción, calor de hidratación, corrosión de armaduras y durabilidad de hormigones en iguales condiciones de trabajabilidad -a asentamiento de cono constante-, a edades hasta de un año. Una faceta general de este estudio es que el eîecto combinado de pérdidas al fuego relativamente altas y de grandes finuras de las cenizas volantes utilizadas ha dado lugar a elevadas exigencias de agua, y tanto más cuanto mayor es la proporción de las cenizas como sustitutivo parcial del cemento.

Hasta la fecha los resultados han demostrado que las resistencias de los hormigones con cenizas son casi iguales, o presentan reducciones aproximadas del 15 por ciento a 28 días, dependiendo de la naturaleza - composición-y finura de las cenizas sustituyentes: con cenizas más ricas en $\mathrm{SiO}_{2}$ y $\mathrm{Al}_{2} \mathrm{O}_{3}$ y más pobres en $\mathrm{CaO}, \mathrm{MgO}, \mathrm{SO}_{3}$, pérdida al fuego y superficie específica en el primer caso; siendo válido lo contrario en el segundo caso. De cualquier modo la resistencia a compresión del hormigón resulta ser tanto mayor cuanto más finas son las cenizas. Las disminuciones de resistencias en el caso de los cementos que las contienen parecen ser menores a medida que la edad de los hormigones aumenta de 7 a 28 días.

En lo que se refiere a la retracción, ésta depende también de la naturaleza de la ceniza volante, tanto añadida en la hormigonera como contenida en el cemento, siendo el efecto directamente proporcional a la cantidad de ceniza.

\section{COMENTARIOS FINALES}

La Encuesta de la Comisión VIII del CEB sobre "Hormigón con Contenido Reducido de Cemento" ha sido tenida en cuenta por el CEMBUREAU, y particularmente por su Comité Técnico (10). Además, el interés del CEMBUREAU por temas relacionados con un contenido mínimo de cemento en el hormigón data por lo menos de 1967, a través de su primer Grupo de Estudio (11). 


\section{CONCLUSIONES GENERALES}

Una primera conclusión general de la Encu ssta podría ser la de que el corto número de respuestas en relación con el número de países (personas) consultados no pemite establecer deducciones definitivas con carácter amplio. Y tanto más cuanto que varias de las respuestas son aún dudosas o provisionales y deben ser confirmadas más a fondo, y que algunos de los puntos contenidos en la Encuesta no han sido investigados o contestados.

No obstante, parece haber quedado claro lo siguiente:

5.1. Que el término "cemento" debe ser bi ən definido en general, y particularmente en lo que concierne a la Encuesta.

5.2. Que se debe hacer asimismo una distinción clara entre reducción de cemento en el hormigón por sustitución de una parte del mismo por algunos otros materiales (en lugar del cemento), y adición de otros materiales al hormigón (además del cemento).

5.3. Que la reducción del cemento en el hormigón no es aconsejable, pues es preciso mantener un contenido mínimo de cemento según el tipo de hormigón, y particularmente en hormigones expuestos a ambientes severos o a ataques externos. Que en tales casos se recomienda aumentar el contenido mínimo de cemento. Y que incluso no esta justificado, en general, sustituir una cantidad mayor de un cemento de una categoría resistente media por una cantidad menor (equivalente) de otro cemento de categoría resistente mayor, en hormigones equivalentes, ya que siempre se requiere una cantidad mínima de matriz de pasta de cemento y de mortero con una relación a/c baja (óptima).

5.4. Que las adiciones como tales están justificadas en proporciones dadas, si mejoran las propiedades reológicas del hormigón fresco y/u otras propiedades y comportamientos del hormigón endurecido, con tal de que no sustituyan al cemento y no provoquen un excesivo (o en todo caso inaceptable) aumento de la relación a/c, perjudicando así al comportamiento del hormigón.

5.5. Que es más aconsejable y seguro útilizar cementơs normalizados que contengan adiciones molidas y mezcladas con el clínker en fábricas de cemento, que mezcladas con el cemento en las hormigoneras, bien de obras o bien de centrales preparadoras de hormigón. Que la primera mezcla se puede llamar cemento, porque lo es; y que la segunda no, porque no lo es (véase el punto 5.1.) y puede conducir a riesgos innecesarios.

5.6. Que, en general, las adiciones utilizadas y estudiadas en los países encuestados han sido cenizas volantes (cenizas de carbón pulverizadas) y excepcionalmente polvo de sílice, puzolanas naturales y escorias siderúrgicas -de horno alto-, en distintas proporciones.

5.7. Que el polvo de sílice es un material especial no fácil de homologar con otros materiales puzolánicos naturales o artificiales, y que probablemente es apto para endurecer con cemento o con cal bajo tratamientos higrotérmicos, y para evitar o reducir los efectos expansivos de las reacciones árido-álcalis.

5.8. Que los motivos para utilizar las adiciones al clínker en las fábricas de cemento y/o al cemento en las obras son de naturaleza técnica y/o económica y/o ecológica. 
5.9. Que no existen realmente, en general, restricciones para el uso de tales adiciones. Y que, no obstante, pueden surgir limitaciones a la extensión de su empleo, a causa de una elevada exigencia de agua de amasado (alta relación a/c —véase 5.4.—), de requisitos especiales para la ejecución y el curado del hormigón en el hormigonado en invierno, o de una excesiva disminución del $\mathrm{pH}$ en la pasta de cemento (mayormente de una reducción de la reserva alcalina), induciendo así la corrosión de las armaduras. Y que, sin embargo, en los hormigones que contienen adiciones no se han observado cambios del $\mathrm{pH}$-alcalinidad- ni diferencias en la corrosión o protección de las armaduras, excepto en el caso de hormigones pobres, o no bien proyectados y/o ejecutados y/o curados y/o sometidos a severas condiciones ambientales o medios agresivos —es decir, como sucede en el caso general-.

5.10. Que, en general, no existen ni controles especiales de la resistencia ni restricciones en el uso de métodos acelerados para determinar la resistencia final de los hormigones con una parte del cemento sustituida por adiciones, si b:en tales métodos parecen causar efectos más notorios en Jos hormigones ricos.

5.11. Que no se pueden extraer conclusiones claras en lo que se refiere a la carbonatación y a la acción del hielo sobre los hormigones "sustituidos". Y que, no obstante, no se han detectado en ellos diferencias sustanciales en cuanto a la carbonatación, en comparación con los hormigones ordinarios normales, habiéndose apreciado, en cambio, una resistencia al hielo casi análoga en ambos, aunque dependiendo en todo caso del aire incluido en ellos.

5.12. Que se han registrado resultados contradictorios en cuanto a la fisuración por retracción plástica y que faltan datos sobre retracción restringida y microfisuración interna. Y que, sin embargo, es previsible una mayor retracción plástica cuando e] hormigón contiene grandes cantidades de adiciones puzolánicas, y no es previsible en cambio la microfisuración si se procede a un curado adecuado, a pesar de que las relaciones a/c altas y las bajas resistencias a la tracción favorecen aquélla.

5.13. Que las resistencias mecánicas del hormigón que contiene adiciones, en comparación con el hormigón normal ordinario que no las contiene, son en general menores a edades tempranas, e iguales e incluso mayores a edades más largas, de forma que la reducción de resistencias observada inicialmente disminuye con el tiempo; y que la relación entre las resistencias a tracción y compresión puede ser mayor o menor (menor en el caso del polvo de sílice y de algunas puzolanas y mayor en el caso de otras).

5.14. Que no se han obtenido resultados claros y decisivos en cuanto a la abrasión, si bien parece que podría ser menor a causa de una menor exudación y segregación en el caso de los hormigones que contienen adiciones.

5.15. Que los cementos con adiciones pueden mostrar una mayor resistencia a los sulfatos y a los ácidos a causa de su mayor densidad, siendo mayor el efecto beneficioso de las adiciones en el caso de los cementos ricos en $\mathrm{C}_{3} \mathrm{~A}$.

5.16. Que el empleo de superplastificantes puede ayudar a rebajar la retracción plástica y restringida, a mejorar el comportamiento reológico de los hormigones frescos, y la resistencia al hielo de los hormigones endurecidos que contienen adiciones puzolánicas tales como las cenizas volantes de carbones pulverizados y el polvo de sílice. 


\title{
R E F E R E N C I A
}

\author{
1) Cembureau: P. Dutron. \\ (2) DiNAMARCA: P. NEPPER-ChristianSEN. \\ (3) éspaña: J. Calleja. \\ (4) ALEMANiA, R. F.: H. Hilsdorf. \\ (5) AUSTRIA: P. NISCHER. \\ (6) ALEMANiA, R. F.: H. Weigler. \\ (7) JORDANIA: R. SHARIF. \\ (8) POLONIA: A. BRANDT. \\ (9) GRECIA: T. P. TASSIOS.
}

(10) CEMBUREAU: Comité Técnico. Carta Circular N.0 61 a los miembros de Comité "ad hoc" sobre Durabilidad del Hormigón, Octubre 1980

(11) CEMBUREAU: Grupo de Estudio sobre Ensayos de Cemento y Hormigón. Cartas Circulares a los miembros, Noviembre-Diciembre 1967.

(12) NORUEGA : R. JOHANSEN.

Nota: Los nombres de las personas adscritos a cada país encuestado corresponden a miembros representativos o asociados en la Comisión VIII del CEB: Tecnología y Control de Calidad del Hormigón, según un anexo de la referencia (10), y/o a personas interesadas en el tema que han respondido a la Encuesta preparada y circulada por (12). Este último caso corresponde al menos a (3).

\section{publicaciones del i.e.t.c.c.}

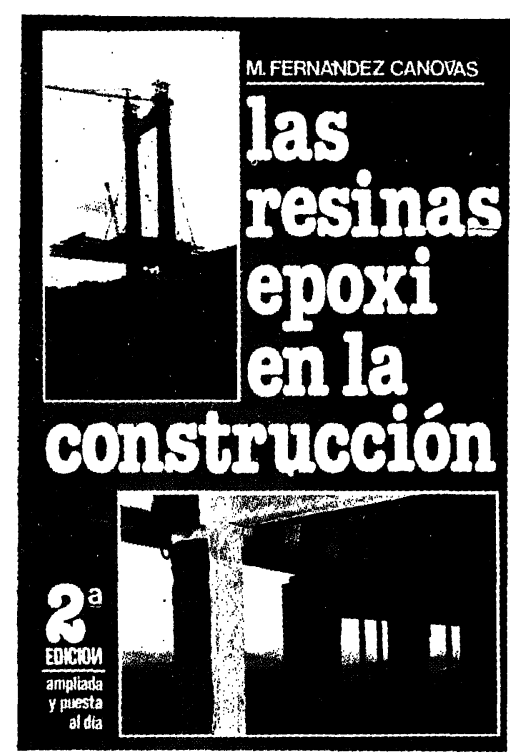

Manuel Fernández Cánovas

Dr. Ingeniero de Construcción

Este libro, el primero en lengua castellana sobre resinas epoxi aplicadas a la construcción, está dirigido a arquitectos, ingenieros, constructores y aplicadores. En él, sobre una reducida base teórica imprescindible, se asienta toda una extensa gama de aplicaciones de gran interés.

El autor trabaja desde hace muchos años en el campo de la investigación, especialmente en el estudio de refuerzos y reparaciones estructurales realizados con resinas epoxi.

Con un lenguaje sencillo se tocan todos los problemas que pueden presentarse en la construcción y en los que la solución puede radicar en el correcto empleo de las resinas epoxi.

Se estudian los componentes de las formulaciones epoxi, sus propiedades fisicas y quimicas, y aplicaciones, deteniéndose, detalladamente, en las siguientes:

Unión de hormigón fresco a hormigón endurecido. - Unión de hormigones entre si. - Inyecciones de fisuras y grietas.... Unión de acero a hormigón. - Barnices y pinturas. - Las combinaciones brea-epoxi. -- Revestimientos de depósitos alimenticios. -.. Sellado de superficies cerámicas. - Protección de tubos. - Los suelos epoxi en sus diferentes variantes. - - Terrazo epoxi.-- Reparación de baches. - Reparación de desperfectos en estructuras. - Reparación de carreteras de hormigón. - Juntas elásticas. - Guardacantos de tableros de puentes. - Refuerzos 'de pilares, vigas, forjados y zapatas, etc. -..- Consolidación de suelos. - Anclajes. - Protección de aceros en pretensado.

Se termina con unos capitulos dedicados a la limpieza y preparación de las superficies según los materiales a unir al control del estado superficial de éstos; a las condiciones de temperatura de aplicación; limpieza de los útiles de trabajo; precauciones en el manejo de los sistemas; almacenaje, mezcla y manejo de las formulaciones epoxi y métodos de ensayo de sistemas y aplicaciones epoxidicas.

Un volumen encuadernado en cartoné plastificado con lomo de tela, de $17 \times 24 \mathrm{~cm}$, compuesto de 334 páginas y 158 figuras y fotografias.

Madrid, 1981.

Precios: España, 1.700 ptas.: extranjero, \$ USA 34.00 